March 31, 2010

OU-HET 660/2010

\title{
Stable Higgs Bosons - new candidate for cold dark matter ${ }^{1}$ -
}

\author{
Yutaka Hosotani \\ Department of Physics, Osaka University, Toyonaka, Osaka 560-0043, Japan
}

\begin{abstract}
The Higgs boson is in the backbone of the standard model of electroweak interactions. It must exist in some form for achieving unification of interactions. In the gauge-Higgs unification scenario the Higgs boson becomes a part of the extradimensional component of gauge fields. The Higgs boson becomes absolutely stable in a class of the gauge-Higgs unification models, serving as a promising candidate for cold dark matter in the universe. The observed relic abundance of cold dark matter is obtained with the Higgs mass around $70 \mathrm{GeV}$. The Higgs-nucleon scattering cross section is found to be close to the recent CDMS II and XENON10 bounds in the direct detection of dark matter. In collider experiments stable Higgs bosons are produced in a pair, appearing as missing energies and momenta so that the way of detecting Higgs bosons must be altered.
\end{abstract}

Keywords: dark matter, Higgs boson, gauge-Higgs unification, Hosotani mechanism PACS: $11.10 . \mathrm{Kk}, 12.60 .-\mathrm{i}, 95.35 .+\mathrm{d}$

\section{Introduction}

What constitutes the dark matter in the universe? Will the Higgs bosons be found at Tevatron and LHC as described in the standard model of electroweak interactions? These are two of the most urgent and important questions in current physics. The dark matter and the Higgs boson, we do not know what they really are. In this talk I would like to present a new scenario that these two mystery particles are really the same. [1, 2]

In the gauge-Higgs unification scenario the 4D Higgs boson is identified with a part of the gauge field in an extra dimension of spacetime. [3]- 6] When the extra dimensional space is not simply connected, the $4 \mathrm{D}$ Higgs boson becomes absolutely stable under a certain conditions. As a consequence Higgs bosons become dark matter of the universe. The relic abundance of the Higgs bosons in the universe is estimated with the Higgs boson mass $m_{H}$ being an only relevant free parameter. The WMAP data for the mass density of cold dark matter is reproduced with $m_{H} \sim 70 \mathrm{GeV}$. [7] The light Higgs boson mass does not contradict with the LEP2 bound $m_{H}>114 \mathrm{GeV}$. In the gauge-Higgs unification scenario the $Z Z H$ coupling vanishes so that the process $e^{+} e^{-} \rightarrow Z \rightarrow Z H$ does not take place, the LEP2 bound thus being evaded.

\footnotetext{
${ }^{1}$ To appear in the Proceedings of "The 10th. International Symposium on Origin of Matter and Evolution of the Galaxies" (OMEG10), March 8-10, 2010, RCNP, Osaka, Japan.
} 


\section{Why do we need "Higgs"?}

The standard model of strong and electroweak interactions is extremely successful. The framework of gauge interactions, in both strong and electroweak interactions, has been firmly established to high accuracy. In the electroweak sector, however, there must be an additional particle yet to be discovered. It is the Higgs boson. Without it the electroweak unification cannot be achieved in the standard model.

Why do we need the Higgs boson? In the unification scenario the theory has larger symmetry than the observed world. The electroweak unified theory has $S U(2)_{L} \times U(1)_{Y}$ gauge symmetry, whereas the only $U(1)_{\text {EM }}$ gauge symmetry, the Maxwell electromagnetic gauge invariance, is observed at low energies. The electroweak $S U(2)_{L} \times U(1)_{Y}$ gauge symmetry must be spontaneously broken to the electromagnetic $U(1)_{\text {EM }}$ gauge symmetry. In the standard model this symmetry breaking is induced by dynamics of the Higgs boson $\phi$.

The Higgs boson develops a vacuum expectation value $\langle\phi(x)\rangle=v$ as a result of the spontaneous symmetry breaking. Its fluctuation $H(x)$ in $\phi(x)=v+H(x)$ is the Higgs boson to be discovered. The Higgs field couples to quarks and leptons with $y(v+H) \bar{\psi} \psi$ where $y$ stands for a Yukawa coupling. Non-vanishing $v$ generates a fermion mass $m=y v$. It implies that the Higgs boson decays into a fermion-antifermion pair with a coupling $y=m / v$. The Higgs boson becomes necessarily unstable in the standard model.

The Higgs boson has not been found yet. We have measured neither the Yukawa couplings nor the Higgs couplings to $W$ and $Z$. We do not have direct information on the Higgs sector.

Unlike the gauge sector the Higgs sector in the standard model does not have a principle governing the interactions. All the couplings of the Higgs boson are arbitrary. They are determined to fit the experimental data. Further it is known that the Higgs boson mass is unstable against radiative corrections. These are unsatisfactory features in the standard model.

\section{Gauge-Higgs unification}

The gauge-Higgs unification scenario gives a solution to those problems. The 4D Higgs field is unified with 4D gauge fields in gauge theory in higher dimensions a la KaluzaKlein. The gauge principle governs the Higgs interactions.

The four-dimensional components $A_{\mu}$ of the gauge potential $A_{M}$ contain $4 \mathrm{D}$ gauge fields, whereas the extra-dimensional component $A_{y}$ contains the $4 \mathrm{D}$ Higgs field.

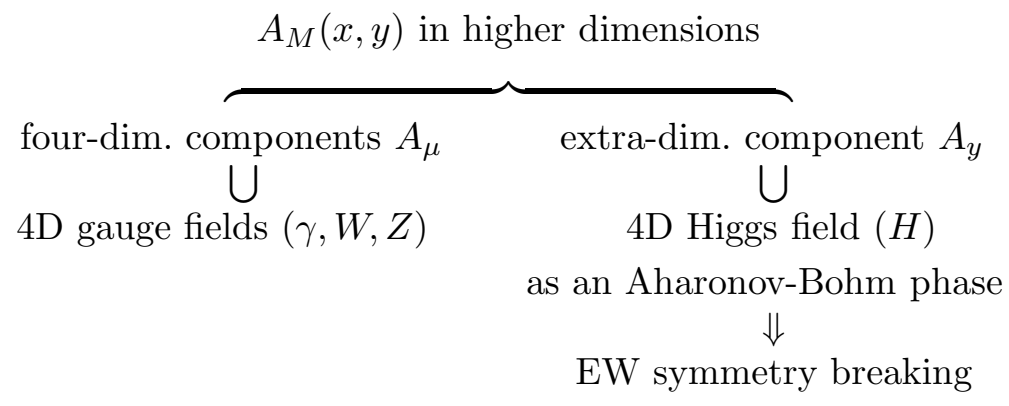


The scenario works well when the extra-dimensional space is non-simply-connected. 3 In that case there appears an Aharonov-Bohm (AB) phase, which is given by the phase of the path-ordered integral along a non-contractible loop $C$ in the extra dimension

$$
P \exp \left\{i g \int_{C} d y A_{y}(x, y)\right\} \text {. }
$$

The $\mathrm{AB}$ phase is a physical degree of freedom remaining in configurations with vanishing field strengths $F_{M N}=0$. The $4 \mathrm{D}$ neutral Higgs field is nothing but $4 \mathrm{D}$ fluctuation of the relevant $\mathrm{AB}$ phase $\theta_{H}$. At the classical level the value of $\theta_{H}$ is undetermined as $F_{M N}=0$ so that the Higgs field is massless. Quantum corrections make the effective potential $V_{\text {eff }}\left(\theta_{H}\right)$ nontrivial. When $V_{\text {eff }}$ is minimized at a nontrivial $\theta_{H} \neq 0$, the gauge symmetry is spontaneously broken. At the same time a finite Higgs mass $m_{H}$ is dynamically generated. This should be contrasted to the earlier attempt of gauge-Higgs unification on $M^{4} \times S^{2}$, in which all $W, Z$, and Higgs bosons have masses of the Kaluza-Klein mass scale $m_{\mathrm{KK}} \cdot[8]$ [1]

A realistic, promising model is constructed with $S O(5) \times U(1)$ gauge symmetry in the five-dimensional Randall-Sundrum (RS) warped spacetime. [12]-[15] The metric of the RS spacetime [16] is given by

$$
d s^{2}=e^{-2 \sigma(y)} \eta_{\mu \nu} d x^{\mu} d x^{\nu}+d y^{2},
$$

where $\eta_{\mu \nu}=\operatorname{diag}(-1,1,1,1), \sigma(y)=\sigma(y+2 L)$, and $\sigma(y)=k|y|$ for $|y| \leq L$. The fundamental region in the fifth dimension is given by $0 \leq y \leq L$. The Planck and $\mathrm{TeV}$ branes are located at $y=0$ and $y=L$, respectively. The bulk region $0<y<L$ is anti-de Sitter spacetime with a cosmological constant $\Lambda=-6 k^{2}$. The metric is specified with two parameters, the AdS curvature scale $k$ and the warp factor $w_{L}=e^{k L} \gg 1$.

The RS spacetime has the same topology as the orbifold $M^{4} \times\left(S^{1} / Z_{2}\right)$. The spacetime points $\left(x^{\mu}, y\right),\left(x^{\mu}, y+2 L\right)$, and $\left(x^{\mu},-y\right)$ are identified with each other. Gauge potentials $A_{M}(x, y)$ are defined in covering space $-\infty<y<\infty$. $A_{M}(x,-y)$ need not be the same as $A_{M}(x, y)$ in gauge theory . It may be twisted by a gauge transformation. Only physical quantities must be single-valued at $y, y+2 L$, and $-y$. This brings orbifold boundary conditions at the Planck and $\mathrm{TeV}$ branes, which, in the model under consideration, break $S O(5) \times U(1)$ to $S O(4) \times U(1) \simeq S U(2)_{L} \times S U(2)_{R} \times U(1)$.

Fermions in the bulk (between the two branes) belong to the vector representation of $S O(5)$. Two vector multiplets are necessary to describe, say, top and bottom quarks. In addition, brane fermions are introduced on the Planck brane, in the $\left(\frac{1}{2}, 0\right)$ representation of $S U(2)_{L} \times S U(2)_{R}$. A brane scalar on the Planck brane, in the $\left(0, \frac{1}{2}\right)$ representation, spontaneously breaks $S U(2)_{R} \times U(1)$ to $U(1)_{Y}$, simultaneously generating mass couplings between the bulk and brane fermions. The surviving symmetry is the standard electroweak (EW) symmetry $S U(2)_{L} \times U(1)_{Y}$.

There appear four AB phases, three of which are absorbed by $W$ and $Z$ bosons. The remaining $\mathrm{AB}$ phase $\theta_{H}$ corresponds to the physical $4 \mathrm{D}$ neutral Higgs boson $H(x)$. It appears in the combination

$$
\hat{\theta}_{H}(x)=\theta_{H}+\frac{H(x)}{f_{H}}, \quad f_{H}=\frac{2}{g_{A}} \sqrt{\frac{k}{z_{L}^{2}-1}} \sim \frac{2}{\sqrt{k L}} \frac{m_{\mathrm{KK}}}{\pi g} .
$$




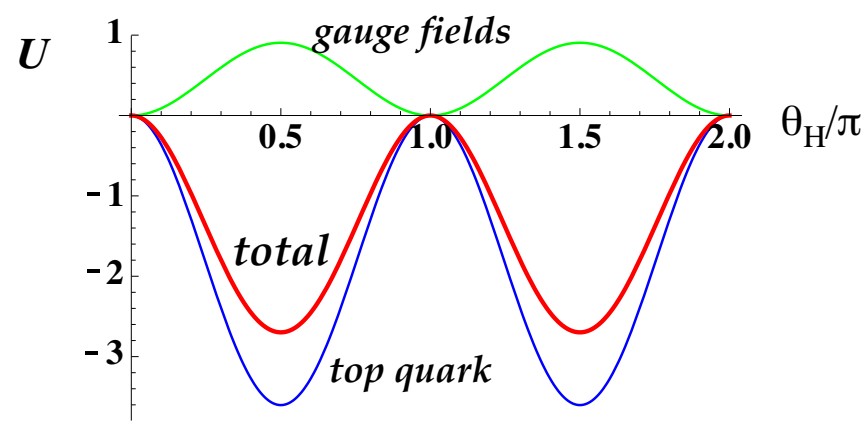

Figure 1: Effective potential $V_{\mathrm{eff}}\left(\theta_{H}\right)=m_{\mathrm{KK}}^{4} /\left(16 \pi^{4}\right) U\left(\theta_{H}\right)$ with $z_{L}=10^{10}$. At its gobal minimum $\theta_{H}=\frac{1}{2} \pi$, the EW symmetry is spontaneously broken to $U(1)_{\mathrm{EM}}$.

Here the Kaluza-Klein (KK) mass scale is $m_{\mathrm{KK}}=\pi k z_{L}^{-1}$, and the $S O(5)$ gauge coupling $g_{A}$ is related to the $4 \mathrm{D} S U(2)_{L}$ weak coupling $g$ by $g=g_{A} / \sqrt{L}$. The theory is invariant under $\theta_{H} \rightarrow \theta_{H}+2 \pi$ as a result of the large gauge invariance.

The value of $\theta_{H}$ is determined by the location of the global minimum of the effective potential $V_{\text {eff }}\left(\theta_{H}\right)$. $V_{\text {eff }}\left(\theta_{H}\right)$ at the one loop level is depicted in fig. 1. It has global minima at $\theta_{H}= \pm \frac{1}{2} \pi$. The contribution from top quarks dominates over those from gauge fields. Contributions from other quarks and leptons are negligible. At $\theta_{H}= \pm \frac{1}{2} \pi$ the EW symmetry $\left(S U(2)_{L} \times U(1)_{Y}\right)$ breaks down to $U(1)_{\mathrm{EM}}$.

\section{Effective theory}

In the effective theory of low energy fields the Higgs field $H(x)$ enters always in the combination of $\hat{\theta}_{H}(x)$ in (4). The effective Lagrangian must be invariant under $\hat{\theta}_{H}(x) \rightarrow$ $\hat{\theta}_{H}(x)+2 \pi$. The Higgs interactions with the $W, Z$ bosons, quarks and leptons at low energies are summarized as [7, 17, 18]

$$
\mathcal{L}_{\mathrm{eff}}=-V_{\mathrm{eff}}\left(\hat{\theta}_{H}\right)-m_{W}^{2}\left(\hat{\theta}_{H}\right) W_{\mu}^{\dagger} W^{\mu}-\frac{1}{2} m_{Z}^{2}\left(\hat{\theta}_{H}\right) Z_{\mu} Z^{\mu}-\sum_{a, b} m_{a b}^{F}\left(\hat{\theta}_{H}\right) \bar{\psi}_{a} \psi_{b}
$$

The effective potential $V_{\text {eff }}\left(\hat{\theta}_{H}\right)$ arises at the one loop level. The mass functions $m_{W, Z}\left(\hat{\theta}_{H}\right)$ and $m_{a b}^{F}\left(\hat{\theta}_{H}\right)$, on the other hand, appear at the tree level.

In the $S O(5) \times U(1)$ model under consideration these mass functions are found, to good accuracy in the warped space, to be [17, 19, 20]

$$
\begin{aligned}
& m_{W}\left(\hat{\theta}_{H}\right) \sim \cos \theta_{W} m_{Z}\left(\hat{\theta}_{H}\right) \sim \frac{1}{2} g f_{H} \sin \hat{\theta}_{H} \quad, \quad\left[\frac{1}{2} g(v+H) \text { in SM }\right], \\
& m_{a b}^{F}\left(\hat{\theta}_{H}\right) \sim y_{a b}^{F} f_{H} \sin \hat{\theta}_{H}, \quad\left[y_{a b}^{F}(v+H) \text { in } \mathrm{SM}\right] .
\end{aligned}
$$

We have listed the formulas in the standard model in brackets. It is seen that $v+H$ in the standard model is replaced approximately by $f_{H} \sin \hat{\theta}_{H}$ in the gauge-Higgs unification. 
The masses of the $W, Z$ bosons and fermions are given by $m_{W}=\frac{1}{2} g f_{H} \sin \theta_{H}$, $m_{Z}=m_{W} / \cos \theta_{W}$, and $m_{a b}^{F}=y_{a b}^{F} f_{H} \sin \theta_{H}$. The Higgs couplings to $W, Z$ and fermions deviate from those in the standard model. It follows from ([6) that

$$
\begin{aligned}
\left(\begin{array}{c}
W W H \\
Z Z H \\
\text { Yukawa }
\end{array}\right) & =\mathrm{SM} \times \cos \theta_{H}, \\
\left(\begin{array}{c}
W W H H \\
Z Z H H
\end{array}\right) & =\mathrm{SM} \times \cos 2 \theta_{H} .
\end{aligned}
$$

As shown in the previous section $\theta_{H}=\frac{1}{2} \pi$ is dynamically chosen so that $f_{H} \sim 246 \mathrm{GeV}$. All of the $W W H, Z Z H$ and Yukawa couplings vanish at $\theta_{H}=\frac{1}{2} \pi$, implying that the Higgs boson cannot decay. This is an exact result as shown below.

\section{Absolutely Stable Higgs bosons}

One of the astonishing results from the gauge-Higgs unification scenario is that Higgs bosons become absolutely stable. It follows from symmetry and dynamics of the theory.

First there exists the mirror reflection symmetry in the fifth dimension; $\left(x^{\mu}, y\right) \rightarrow$ $\left(x^{\mu},-y\right)$. It implies that physics is invariant under

$$
\hat{\theta}_{H}(x)=\theta_{H}+\frac{H(x)}{f_{H}} \rightarrow-\hat{\theta}_{H}(x)
$$

while all other SM particles remain unchanged.

Secondly there arises enhanced gauge invariance. In our model the bulk fermions are all in the vector representation of $S O(5)$. There is no matter field in the spinor representation of $S O(5)$. Brane fermions are introduced at the Planck brane only. In this case the theory turns out invariant under

$$
\theta_{H} \rightarrow \theta_{H}+\pi
$$

All physical quantities become periodic in $\theta_{H}$ with a reduced period $\pi$.

Now recall that the dynamics select $\theta_{H}=\frac{1}{2} \pi$. Then we have equivalence relations

$$
\frac{\pi}{2}+\frac{H}{f_{H}} \Leftrightarrow-\frac{\pi}{2}-\frac{H}{f_{H}} \Leftrightarrow \frac{\pi}{2}-\frac{H}{f_{H}}
$$

where the first and second equivalece relations follow from (8) and (9), respectively. The net result is the change in the sign of the 4D Higgs field $H(x)$. All other SM fields remain unchanged. There emerges the $H$-parity invariance.

$$
\begin{aligned}
& \text { Higgs boson : H-parity - } \\
& \text { all other SM particles : H-parity }+ \text {. }
\end{aligned}
$$

Among low energy fields only the Higgs field is $H$-parity odd. The Higgs boson becomes absolutely stable, protected by the $H$-parity conservation. 


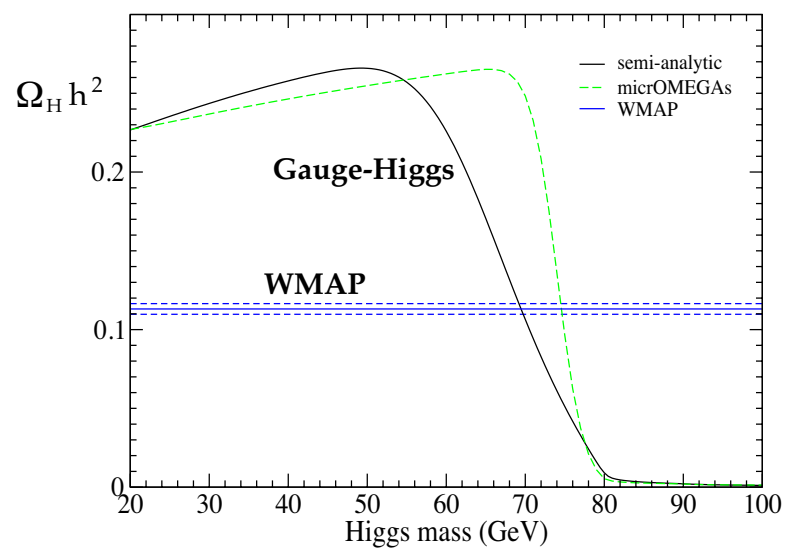

Figure 2: Relic abundance of the Higgs dark matter. The solid curve is obtained by the semi-analytic formulas. The horizontal band is the WMAP data $\Omega_{\mathrm{CDM}} h^{2}=0.1131 \pm$ 0.0034 .

\section{Higgs dark matter}

As an important consequence, abosolutely stable Higgs bosons become cold dark matter (CDM) in the present universe. They are copiously produced in the very early universe. As the annihilation rates of Higgs bosons fall below the expansion rate of the universe, the annihilation processes get effectively frozen and the remnant Higgs bosons become dark matter. [7]

In the gauge-Higgs unification scenario the annihilation rates can be estimated from the effective Lagrangian (5) with (6), once the Higgs boson mass $m_{H}$ is given. 21, 22, If $m_{H}>m_{W}$, the dominant annihilation modes are $H H \rightarrow W W, Z Z$. The rate is large so that the resultant relic abundance becomes very small. For $m_{H}<m_{W}$ the relevant annihilation modes are $H H \rightarrow W^{*} W^{*}, Z^{*} Z^{*}, b \bar{b}, c \bar{c}, \tau \bar{\tau}$, and $g g$. Here $W^{*}\left(Z^{*}\right)$ denotes virtual $W(Z)$ which subsequently decays into a fermion pair. Annihilation into a gluon $(g)$ pair takes place through a top quark loop.

The relic abundance of Higgs bosons as a function of $m_{H}$ is depicted in fig. $2, \Omega_{H} h^{2}$ determined from the WMAP data is reproduced with $m_{H} \sim 70 \mathrm{GeV}$. With $m_{H}=70 \mathrm{GeV}$, the freeze-out temperature is $T_{f} \sim 3 \mathrm{GeV}$. The relative contributions of the $H H \rightarrow$ $W^{*} W^{*}$ and $b \bar{b}$ modes are $61 \%$ and $34 \%$, respectively.

The Higgs dark matter can be detected by observing Higgs-nucleon elastic scattering $H N \rightarrow H N$. The spin-independent (SI) Higgs-nucleon scattering cross section is found to be

$$
\sigma_{\mathrm{SI}} \simeq \frac{1}{4 \pi}\left(\frac{2+7 f_{N}}{9}\right)^{2} \frac{m_{N}^{4}}{f_{H}^{4}\left(m_{H}+m_{N}\right)^{2}},
$$

where $f_{N}=\sum_{q=u, d, s}\left\langle N\left|m_{q} \bar{q} q\right| N\right\rangle / m_{N}$ is estimated in a range $0.1 \sim 0.3$. With $m_{H}=$ $70 \mathrm{GeV}$ and $f_{H}=246 \mathrm{GeV}, \sigma_{\mathrm{SI}}$ is estimated to be $\sigma_{\mathrm{SI}} \simeq(1.2 \sim 2.7) \times 10^{-43} \mathrm{~cm}^{2}$.

The present experimental upper bounds for the spin-independent WIMP-nucleon cross sections come from CDMS II 23] and XENON10 [24]. From the recent CDMS II data $\sigma_{\mathrm{SI}} \lesssim 7 \times 10^{-44} \mathrm{~cm}^{2}$ at $90 \% \mathrm{CL}$ with the WIMP mass $70 \mathrm{GeV}$. With many 
uncertainties and ambiguity in the analysis taken into account, this does not necessarily mean that the present gauge-Higgs unification model is excluded. The next generation experiments for the direct detection of WIMP-nucleon scattering are awaited to pin down the rate.

The scenario under discussion, in which the Higgs bosons responsible for the EW symmetry breaking become the dark matter, differs from that of refs. [25, 26] where an additional Higgs doublet with odd parity is introduced by hand. It also differs from the Kaluza-Klein (KK) dark matter scenario in which additional fields with odd KK parity become dark matter.[27]-[31] Unlike those models the relic abundance and the direct detection rate are unambiguously estimated in the present model once $m_{H}$ is known.

\section{At colliders}

Higgs bosons are produced in pairs at colliders. Typical production processes are $Z^{*} \rightarrow$ $Z H H, W^{*} \rightarrow W H H, W W \rightarrow H H, Z Z \rightarrow H H$, and $g g \rightarrow H H$. Higgs bosons are stable so that they appear as missing energies and momenta. The appearance of two particles of missing energies and momenta in the final state makes experiments hard, but not impossible. These events must be distinguished from those involving neutrinos. In all of the experiments performed so far, Higgs bosons were searched by trying to identify their decay products. Since Higgs bosons are stable, this way of searching Higgs bosons must be altered.

There arise deviations in the gauge couplings of quarks and leptons, resulting in violation of the universality. [15] The deviations in the $W$ and $Z$ couplings are tiny $(0.1 \%$ to $1 \%)$ except for top quarks. An important prediction is given for the forward-backward asymmetry in the $e^{+} e^{-}$collisions on the $Z$ pole. 32] CP violation, anomalous magnetic moment, and electric dipole moment in gauge-Higgs unification have been also discussed. [33]

\section{Summary}

In the gauge-Higgs unification scenario the Higgs boson is unified with gauge fields. The Higgs bosons naturally become stable in the $S O(5) \times U(1)$ unification. They become the cold dark matter of the present universe. The mass of the Higgs boson is determined to be $\sim 70 \mathrm{GeV}$ from the WMAP data. The direct detection rate for the WIMP(Higgs)-nucleon scattering is predicted to be very close to the current limit.

In collider experiments Higgs bosons appear as missing energies and momenta so that the way of searching Higgs bosons must be altered. The gauge-Higgs unification scenario also leads to slight deviations from the standard model in the electroweak gauge couplings, which can be checked in experiments.

To summarize, the gauge-Higgs unification scenario is promising. The stable Higgs boson is a new candidate for cold dark matter.

\section{Acknowledgment}

This work was supported in part by Scientific Grants from the Ministry of Education and Science, Grant No. 20244028, Grant No. 20025004, and Grant No. 50324744. 


\section{References}

[1] Y. Hosotani, arXiv:1003.3129 [hep-ph].

[2] Y. Hosotani, AIP Conf. Proc. 1078, 150 (2009) arXiv:0809.2181 [hep-ph]].

[3] Y. Hosotani, Phys. Lett. B 126, 309 (1983).

[4] Y. Hosotani, Annals Phys. 190, 233 (1989).

[5] A. T. Davies and A. McLachlan, Phys. Lett. B 200, 305 (1988); Nucl. Phys. B 317, 237 (1989).

[6] H. Hatanaka, T. Inami and C. S. Lim, Mod. Phys. Lett. A 13, 2601 (1998) arXiv:hep-th/9805067.

[7] Y. Hosotani, P. Ko and M. Tanaka, Phys. Lett. B 680, 179 (2009) arXiv:0908.0212 [hep$\mathrm{ph}]$.

[8] D. B. Fairlie, Phys. Lett. B 82, 97 (1979); J. Phys. G 5, L55 (1979).

[9] N. S. Manton, Nucl. Phys. B 158, 141 (1979).

[10] S. Randjbar-Daemi, A. Salam and J. Strathdee, Nucl. Phys. B 214, 491 (1983).

[11] Y. Hosotani, Phys. Lett. B 129, 193 (1984); Phys. Rev. D 29, 731 (1984).

[12] K. Agashe, R. Contino and A. Pomarol, Nucl. Phys. B 719, 165 (2005) arXiv:hep-ph/0412089.

[13] A. D. Medina, N. R. Shah and C. E. M. Wagner, Phys. Rev. D 76, 095010 (2007) arXiv:0706.1281 [hep-ph]].

[14] Y. Hosotani, K. Oda, T. Ohnuma and Y. Sakamura, Phys. Rev. D 78, 096002 (2008) [Erratum-ibid. D 79, 079902 (2009)] arXiv:0806.0480 [hep-ph]].

[15] Y. Hosotani, S. Noda, and N. Uekusa, arXiv:0912.1173 [hep-ph] (to appear in Prog. Theoret. Phys.).

[16] L. Randall and R. Sundrum, Phys. Rev. Lett. 833370 (1999).

[17] Y. Hosotani and Y. Kobayashi, Phys. Lett. B 674, 192 (2009) arXiv:0812.4782 [hep-ph]].

[18] Y. Sakamura, Phys. Rev. D 76, 065002 (2007) arXiv:0705.1334 [hep-ph]].

[19] Y. Sakamura and Y. Hosotani, Phys. Lett. B 645, 442 (2007) arXiv:hep-ph/0607236.

[20] Y. Hosotani and Y. Sakamura, Prog. Theor. Phys. 118, 935 (2007) arXiv:hep-ph/0703212.

[21] G. Jungman, M. Kamionkowski and K. Griest, Phys. Rep. 267, 195 (1996).

[22] G. Bertone, D. Hooper and J. Silk, Phys. Rep. 405, 279 (2005).

[23] Z. Ahmed et al. [CDMS Collaboration], Phys. Rev. Lett. 102, 011301(2009) arXiv:0802.3530 [astro-ph]]; arXiv:0912.3592 [astro-ph.CO].

[24] J. Angle et al. [XENON Collaboration], Phys. Rev. Lett. 100, 021303(2008) arXiv:0706.0039 [astro-ph]].

[25] R. Barbieri, L.J. Hall and V.S. Rychkov, Phys. Rev. D 74, 015007 (2006).

[26] M. Gustafsson, E. Lundström, L. Bergström and J. Edsjö, Phys. Rev. Lett. 99, 041301 (2007).

[27] K. Agashe, A. Falkowski, I. Low and G. Servant, JHEP 0804, 027 (2008) arXiv:0712.2455 [hep-ph]].

[28] M. Regis, M. Serone and P. Ullio, JHEP 0703, 084 (2007) arXiv:hep-ph/0612286.

[29] G. Panico, E. Ponton, J. Santiago and M. Serone, Phys. Rev. D 77, 115012 (2008) arXiv:0801.1645 [hep-ph]].

[30] M. Carena, A. D. Medina, N. R. Shah and C. E. M. Wagner, Phys. Rev. D 79, 096010 (2009) arXiv:0901.0609 [hep-ph]].

[31] N. Haba, S. Matsumoto, N. Okada and T. Yamashita, arXiv:0910.3741 [hep-ph].

[32] N. Uekusa, arXiv:0912.1218 [hep-ph].

[33] Y. Adachi, C. S. Lim and N. Maru, Phys. Rev. D 76, 075009 (2007) arXiv:0707.1735 [hep-ph]]; Phys. Rev. D 80, 055025 (2009) [arXiv:0905.1022 [hep-ph]]. 